\title{
Crystal structure of highly concentrated, ionic microgel suspensions studied by small-angle $x$-ray scattering
}

\author{
U. Gasser* \\ Laboratory for Neutron Scattering, ETH Zurich and Paul Scherrer Institut, 5232 Villigen PSI, Switzerland
}

\author{
A. Fernandez-Nieves ${ }^{\dagger}$ \\ School of Physics, Georgia Institute of Technology, Atlanta, Georgia 30332-0430, USA \\ (Received 7 December 2009; revised manuscript received 17 March 2010; published 3 May 2010)
}

\begin{abstract}
We present a small-angle $\mathrm{x}$-ray scattering study of the crystal structure formed by $p \mathrm{H}$-sensitive poly $(2-$ vinylpyridine) microgel particles with 5 wt $\%$ of cross-linker. We focus on highly swollen particles and explore concentrations ranging from below close packing to well above close packing, where the particles are forced to shrink and/or interpenetrate. The crystal structure found from poly- as well as monocrystalline domains is random hexagonally close packed, as also observed in hard spheres.
\end{abstract}

DOI: 10.1103/PhysRevE.81.052401

\section{INTRODUCTION}

Microgel particles are crosslinked polymer particles with diameters in the $\mathrm{nm}$ and $\mu \mathrm{m}$ ranges, which can change their size due to variations in solubility, as induced by changes in temperature [1] and hydrostatic pressure [2], $p \mathrm{H}[3]$, salt concentration [4], or external osmotic pressure [5]. This transition between swollen and deswollen states [6] is very attractive for many applications [7-9] and also for studying the effect of particle softness over the phase behavior and the transition from hard to soft-sphere behavior.

Despite the large interest in the properties of soft and deformable particle-suspensions, their macroscopic behavior is not as well understood as that of hard particles. In addition, the interaction between microgel particles and its dependence on particle concentration is still not known in detail [10]. From a theoretical point of view, charged soft particles are predicted to form crystal structures which are different from those formed by hard spheres at sufficiently high particle concentrations [11]. However, our previous neutron-scattering study on charged microgels [12] as well as experimental results by other groups $[13,14]$ indicate that the crystal structure is comparable to that of hard spheres even for very dense packing, where particles have to deform and/or interpenetrate.

In this paper, we present further insight from small-angle $x$-ray scattering (SAXS) experiments into the crystal structures formed by charged, $p \mathrm{H}$-sensitive microgels up to concentrations that cannot be reached with hard-sphere-like particles, which can only fill space to a maximal volume fraction of $\phi_{c p} \approx 0.74$. We find that the crystal structure remains random hexagonal-close packed (rhcp), a random mixture of face-centered cubic (fcc), and hexagonal-close packed (hcp) consistent with our previous findings [12]. However, in this study the resolution of the SAXS instrument allows us to resolve individual Bragg peaks to obtain direct evidence of the rhcp structure. This crystal structure is commonly observed in hard spheres although their equilib-

\footnotetext{
*urs.gasser@psi.ch

†alberto.fernandez@physics.gatech.edu
}

PACS number(s): 82.70.-y, 64.70.D-

rium structure is the fcc crystal lattice. However, the freeenergy difference between fcc and hcp is so small that rhcp initially forms due to stacking faults between hexagonal lattice planes $[15,16]$.

\section{EXPERIMENTAL METHODS}

\section{A. Experimental system}

As described in more detail in our previous publication [12], we study $p \mathrm{H}$-sensitive poly(2-vinylpyridine) (2VP) particles crosslinked with divinylbenzene (DVB) (5 wt \%). As the crosslinker reacts faster than the 2VP monomer during the polymerization, the particles are expected to have a decaying concentration of crosslinker from the center to the periphery [17]. Their swelling behavior was determined by dynamic light-scattering measurements, which show that the particles swell at $p \mathrm{H} \leq 4$, due to ionization of the $2 \mathrm{VP}$ groups, and reach a maximum hydrodynamic size of $d_{\text {swollen }}=(500 \pm 10) \mathrm{nm}$. During the synthesis [18], polymerization was started with $2,2^{\prime}$-azobis(2-amidinopropane) dihydrochloride, which gives the particles a positive surface charge; this provides the required repulsion between particles to guarantee the colloidal stability of the suspension, especially in their deswollen state at $p \mathrm{H} \gtrsim 4$ [3]. In this deswollen state, the particles were imaged with Transmission Electron Microscopy to confirm the suspension monodispersity and to estimate the collapsed size of the particles, $d_{T E M}$ $=(182 \pm 5) \mathrm{nm}$, where the error corresponds to the standard deviation of the measured size distribution, which is a measure of sample polydispersity.

Samples were kept at $p \mathrm{D}=2.7$ by adding deuterated hydrochloric acid $(\mathrm{DCl})$ to the heavy water solvent $\left(\mathrm{D}_{2} \mathrm{O}\right)$. As the same samples were studied by small-angle neutron scattering (SANS) [12], deuterated acid and water were used and, therefore, we use the abbreviation " $p \mathrm{D}$ " instead of " $p \mathrm{H}$." A very concentrated sample was prepared using a rotary evaporator and dilutions were obtained by adding $\mathrm{D}_{2} \mathrm{O}$ and keeping the $p \mathrm{D}$ constant by adding $\mathrm{DCl}$. As the volume fraction is not a directly accessible quantity in microgel suspensions, we use $\xi=c V_{0}^{c \approx 0}$ as a measure of concentration, where $c$ is the particle number density and $V_{0}^{c \approx 0}$ is the par- 

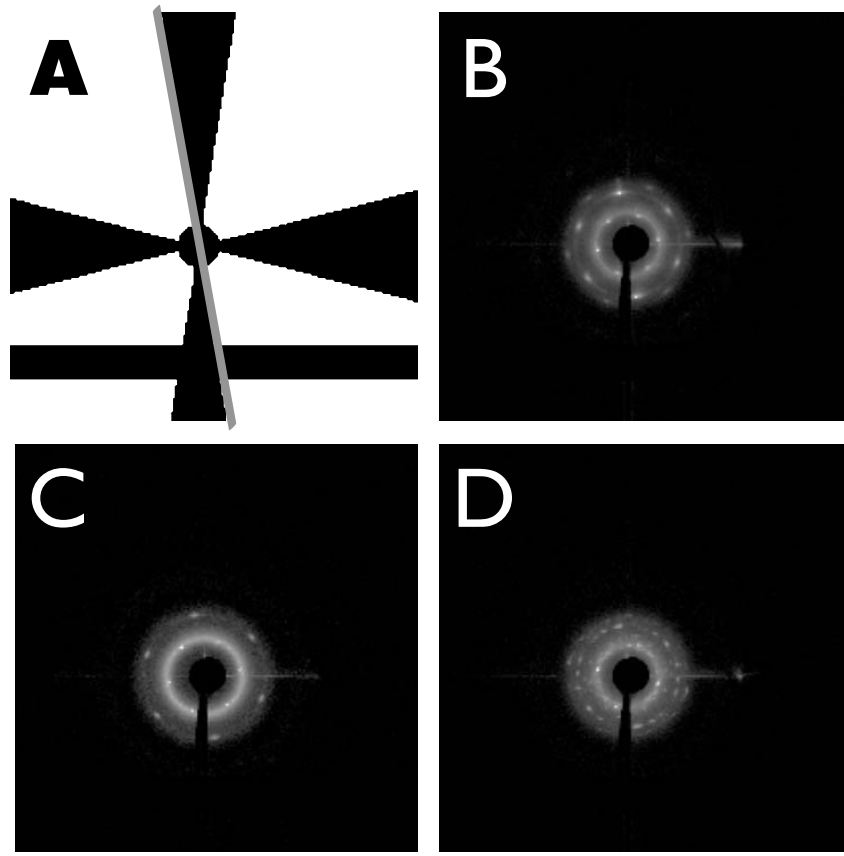

FIG. 1. (a) Detector areas excluded from the calculation of azimuthal averages are shown by the dark regions. The orientation of the sample capillary is indicated by the gray line. The following panels show detector images obtained within $0.1 \mathrm{~s}$ at (b) $\xi=0.63$, (c) $\xi=0.95$, and (d) $\xi=0.55$ with logarithmic gray scale.

ticle volume in the swollen state at very low concentration. We determine $V_{0}^{c \approx 0}$ from the measured particle diameter obtained from dynamic light scattering [12]. Samples with $\xi$ $\geq 0.55$ up to the highest studied concentration of $\xi=1.05$ were found to crystallize within $\sim 1 \mathrm{~h}$ by visual inspection, as the first Bragg peak can be detected with visible light.

\section{B. SAXS}

All samples were measured on the cSAXS beamline of the Swiss Light Source at Paul Scherrer Institut, Switzerland. The instrument was set up for a wavelength of $1.424 \AA$ and a distance of $7.18 \mathrm{~m}$ from sample to detector. The detector had a pixel size of $172 \mu \mathrm{m}$ [19] and the collimation of the beam covering an area of $\approx 200 \times 200 \mu \mathrm{m}^{2}$ on the sample gave a better $q$ resolution than the one obtained earlier in neutron-scattering measurements [12]. The samples were loaded in quartz glass capillaries with an inner diameter of 1 $\mathrm{mm}$ and centered in the beam by a short series of measurements with a point detector. Typical data sets of 50 measurements of $0.1 \mathrm{~s}$ each were taken with the $2 d$ detector. As a consequence of the $\approx 500 \mathrm{~nm}$ particle diameter only the region of $200 \times 200$ pixels close to the direct beam was of interest for determining the form factor and the crystal structure $\left(10^{-3}<q<2 \times 10^{-2} \AA^{-1}\right)$; all the presented analysis was done with this central part of the detector.

Scattering from the horizontal and vertical slits collimating the x-ray beam resulted in an increased background along horizontal and vertical sections. This background was eliminated using a mask excluding the corresponding sections, as shown in Fig. 1(a). Furthermore, the capillary was tilted by $10^{\circ}$ away from the vertical direction to move Bragg peaks appearing along the capillary direction out of the excluded sectors; in several samples the crystals appeared to be oriented along the capillary such that second-order Bragg peaks appear along this direction. The x-ray beam was observed to change the suspension structure within $\approx 10 \mathrm{~s}$ of exposure. In crystalline samples at $\xi \lesssim 0.6$, this resulted in the disappearance of the second- and third-order Bragg peaks and in a clear intensity reduction of the 1st order peaks; for higher $\xi$, the peaks remained but their intensities were diminished. This is most probably due to local heating and/or a local change of $p \mathrm{D}$ due to ionization caused by the $\mathrm{x}$-ray beam. Thus, the crystal structures were analyzed using the first measurement of $0.1 \mathrm{~s}$ in a series and different beam positions on the sample were chosen for repeated measurements on the same sample.

\section{RESULTS AND DISCUSSION}

For $\xi \geq 0.63$, the scattering images generally show Bragg peaks with a sixfold rotational symmetry, as shown in Figs. 1(b) and 1(c). These sharp peaks lie on top of a background due to polycrystalline or fluid domains in the sample, which most probably occupy the central region of the sample capillary. The well visible individual Bragg peaks originate from crystals that have nucleated heterogeneously on the wall of the capillary, which leads to the well defined orientation of hexagonal particle planes perpendicular to the x-ray beam. Due to the sixfold pattern, the crystal must have a sixfold symmetry axis. Furthermore at these high concentrations, the monocrystals on the wall are typically larger than the area of $\approx 200 \times 200 \mu \mathrm{m}^{2}$ illuminated by the beam. The positions of the Bragg peaks at constant $q$ value were determined and are found to be located on a regular hexagon; no distortion of the scattering pattern along any direction was detected, which could have been expected due to the flow along the capillary direction when the sample is filled into the capillary. For $\xi$ $=0.55$, the scattering pattern exhibits rings containing a larger number of Bragg peaks from several crystallites, as shown in Fig. 1(d), indicating that the heterogeneously nucleated crystals are smaller than the beam size and randomly oriented inside the capillary at this lower concentration. This might reflect an increased nucleation density rate, $J$, compared to $\xi>0.55$, consistent with the behavior of hard spheres, which exhibit a decreasing $J$ with increasing volume fraction $\phi \gtrsim 0.56$ due to the reduced particle mobility and the increase of the nucleus surface tension with undercooling [20-22].

To compare the measurements with crystal structures, the structure factor $S(q)=I(q) / F(q)$ was determined from an azimuthal average $I(q)$ of the $2 d$ detector image and the form factor $F(q)$ shown in Fig. 2, which was obtained at $\xi=0.15$. The line represents a fit to the measurement obtained using the particle density profile, $d(r)$, shown in the inset, which is modeled as a superposition of constant functions, $\chi_{j}(r)$, corresponding to small $r$-intervals in the range from the particle center, $r=0$, to the particle radius, $r=260 \mathrm{~nm}$. Explicitly, $d(r)=\sum_{j} c_{j} \chi_{j}(r)$, with 


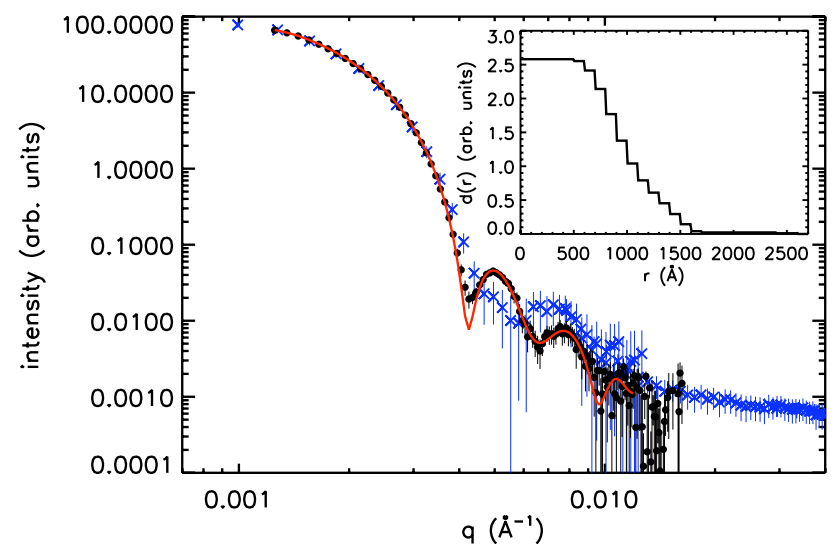

FIG. 2. (Color online) Form factor of $2 \mathrm{VP}$ particles measured by SAXS $(\bullet)$ and SANS $(\times)$ [12] at $\xi=0.15$. The line represents a fit to the SAXS measurement that was obtained from the particle density profile $d(r)$ shown in the inset.

$$
\chi_{j}(r)= \begin{cases}1 & \text { for } r_{j} \leq r<r_{j+1} \\ 0 & \text { otherwise }\end{cases}
$$

and $c_{j}$ the corresponding amplitudes, which we use as fitting parameters. The fit to the measurement is obtained using the Fourier transforms $\hat{\chi}_{j}=\mathcal{F}\left(\chi_{j}\right)$ and $I(q)=\mathcal{S}\left[\left(\Sigma_{j} c_{j} \hat{\chi}_{j}\right)^{2}\right]$, where $\mathcal{S}$ represents a "smearing" of $I(q)$ due to the limited $q$-resolution of the instrument; this is done as a convolution with a Gaussian with width $\sigma=0.03 q$ :

$$
I(q)=\sum_{j} c_{j} \int_{0}^{\infty} d s \hat{\chi}_{j}(q+s) \frac{1}{\sqrt{2 \pi} \sigma} \exp \left(-\frac{s^{2}}{2 \sigma^{2}}\right) .
$$

Consistent with the particle synthesis, the particle density profile used to describe the form-factor measurement reflects that the microgels have a dense core surrounded by a dilute shell. As in Ref. [12], this form factor measured with a dilute sample is an approximation for measurements at high $\xi$. However, as the shrinking and/or deformation of the particles at high concentrations is not understood in detail and is thus hard to be accounted for, we use this form factor for all presented measurements. We also note that the maximum $\xi$ we use in our SAXS measurements is 1.05 , which is not excessively above the close-packing limit for hard spheres. Figure 2 also shows the form factor obtained by SANS, which differs slightly from the SAXS measurement for $q$ $>0.003 \AA^{-1}$ due to the different contrast obtained with neutrons and x-rays, the lower $q$ resolution of the SANS measurement, and perhaps also due to the smaller $p \mathrm{D}$ of the samples during the SAXS experiments $(p \mathrm{D}=2.7)$ compared to that during the SANS measurements $(p \mathrm{D}=3.0)$.
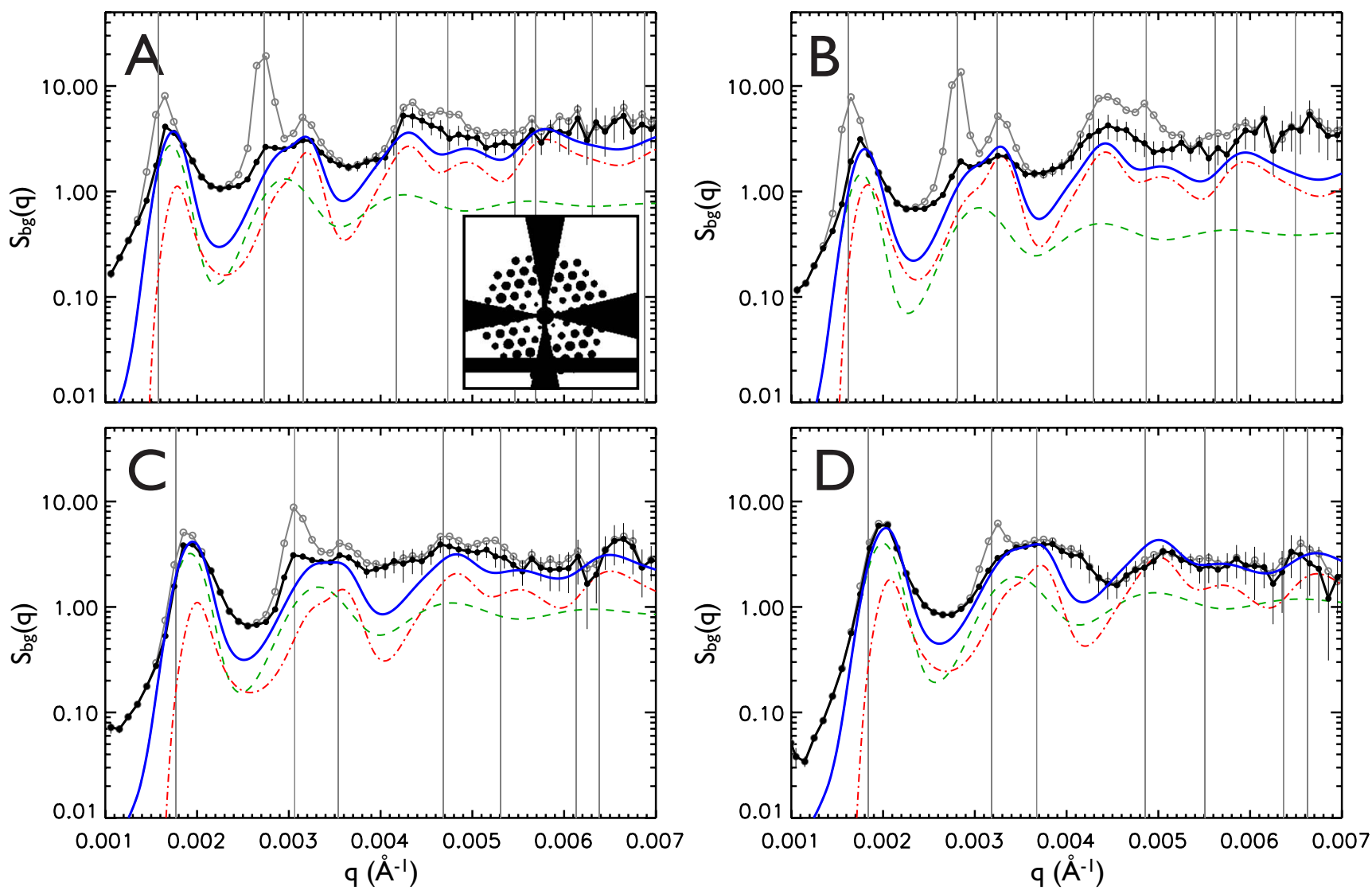

FIG. 3. (Color online) SAXS measurements (-) of the polycrystalline and fluid background at $\xi=0.63$ (a), 0.70 (b), 0.80 (c), and 0.95 (d). The total measurement including the monocrystalline part is shown by the gray circles. The curves represent the fluid contribution (- - ), the polycrystalline contribution (-·), and their sum (-). The thin vertical lines show the peak positions for a hexagonal layer of particles. The inset in (a) shows a typical mask used to obtain the background scattering. 

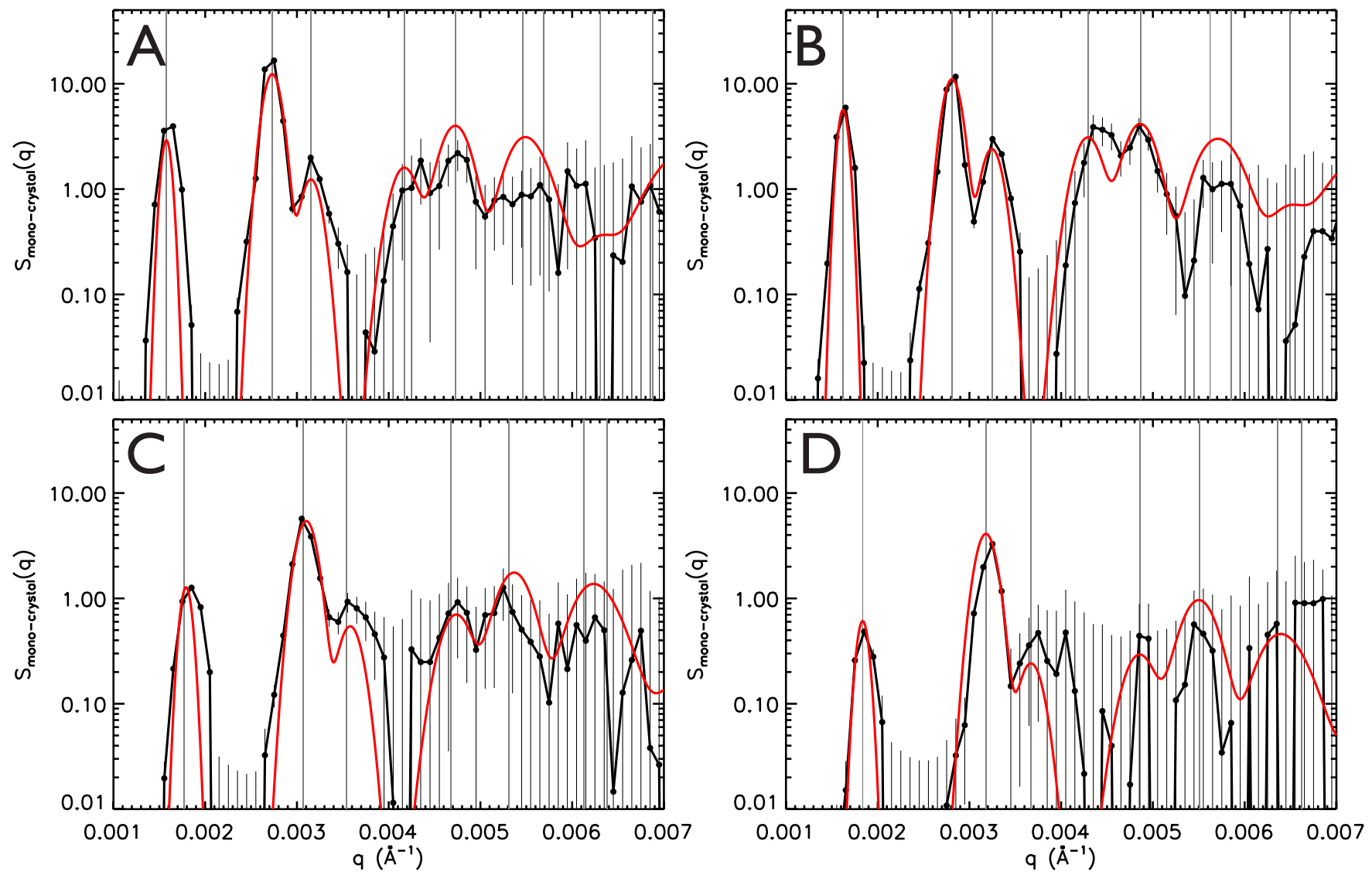

FIG. 4. (Color online) $S_{\text {mono-cryst. }}(q)$ as determined from SAXS measurements ( ) at $\xi=0.63$ (a), 0.70 (b), 0.80 (c), and 0.95 (d). The curves $(-)$ show the rhcp fit to the data. The thin vertical lines show the peak positions for a hexagonal layer of particles.

The hexagonal pattern of Bragg peaks typically observed for $\xi \gtrsim 0.6$ indicates that hexagonal particle planes are oriented perpendicular to the beam. This rules out bcc, fcc, and A15 [23] crystal structures that were also taken into account in Ref. [12], since they do not give a hexagonal pattern of Bragg peaks. The expected structure is, therefore, either hcp or its random counterpart, rhcp, which consists of a random mixture of hcp- and fcc-like stackings of hexagonal planes $[24,25]$. There are three possible positions for hexagonal planes in such a close-packed stacking. Accordingly, there are two possibilities for the vector connecting the centers of two neighboring particles in consecutive hexagonal planes: $\boldsymbol{r}_{1}$ and $\boldsymbol{r}_{2}$. To allow for randomness in the stacking, we use a parameter $\alpha$, which gives the probability that the stacking between the second and the third plane is given by $\boldsymbol{r}_{1}$, if the stacking between the first and the second plane is also given by $\boldsymbol{r}_{1} . \alpha=0$ thus corresponds to pure hcp, $\alpha=1$ to pure fcc, and $\alpha=1 / 2$ to a completely random mixture of hcp and fcc stackings. The structure factor of the rhcp crystal is given by the structure factor of a hexagonal plane, $S_{\text {hex }}(\boldsymbol{q})$, and a factor representing the random stacking of the hexagonal planes

$$
\begin{aligned}
S_{\text {rhcp }}(\boldsymbol{q})= & S_{\text {hex }}(\boldsymbol{q}) \alpha(1-\alpha)\left\{1-\cos \left[\boldsymbol{q} \cdot\left(\boldsymbol{r}_{1}-\boldsymbol{r}_{2}\right)\right]\right\} /\{1-2 \alpha \\
& +3 \alpha^{2}-2 \alpha^{2}\left[\cos \left(\boldsymbol{q} \cdot \boldsymbol{r}_{1}\right)+\cos \left(\boldsymbol{q} \cdot \boldsymbol{r}_{2}\right)\right] \\
& \left.+\alpha^{2} \cos \left[\boldsymbol{q} \cdot\left(\boldsymbol{r}_{1}-\boldsymbol{r}_{2}\right)\right]+(2 \alpha-1) \cos \left[\boldsymbol{q} \cdot\left(\boldsymbol{r}_{1}+\boldsymbol{r}_{2}\right)\right]\right\} .
\end{aligned}
$$

Equation (1) is not valid, if $\alpha=0, \alpha=1$, or if the denominator vanishes. These special cases have to be treated separately as explained in Ref. [24]. The structure factor $S_{\text {hex }}(\boldsymbol{q})$ gives "Bragg rods" oriented along $\hat{\boldsymbol{c}}$, the unit vector perpendicular to the hexagonal planes, while the factor following $S_{\text {hex }}(\boldsymbol{q})$ on the right-hand side of Eq. (1) modulates the intensity of the Bragg rods along this $\hat{\boldsymbol{c}}$ direction. Both broad peaks as well as sharp Bragg peaks can appear along the Bragg rods depending on the value of $\alpha$ and the position of the Bragg rod in $q$ space given by $\boldsymbol{q}-(\boldsymbol{q} \cdot \hat{\boldsymbol{c}}) \hat{\boldsymbol{c}}$, which lies in the $q$ plane of the detector, if $\hat{c}$ is oriented along the beam direction. Among the first rods, Bragg peaks do not appear on the first and third ring of rods at $q=2 \pi / a$ and $q=\pi / a$, respectively [24]; $a$ is the nearest-neighbor distance of the lattice sites.

The scattering from the polycrystalline or fluid background and the monocrystal giving rise to the observed sharp Bragg peaks were taken into account separately. The polycrystalline and fluid background contribution was obtained by masking the strong Bragg peaks arising from the heterogeneously nucleated single crystals, as shown in the inset of Fig. 3(a). Such a mask was determined for each measurement to accurately avoid the Bragg peaks. Using this mask, the azimuthally averaged scattering intensity from the polycrystalline or fluid background is determined and then divided by the form factor to obtain the background structure factor $S_{b g}(q)$ as shown in Fig. 3 for several $\xi$. This is fit using a polycrystalline and a fluid contribution. The former is cal- 


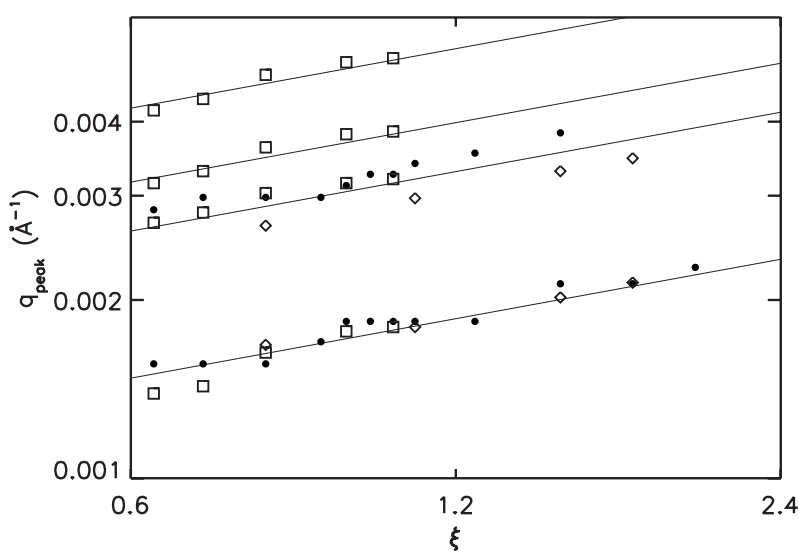

FIG. 5. Peak positions of the first four peaks in $S(q)$ as a function of $\xi$ determined by SAXS $(\square)$, USANS $(\diamond)$, and SANS $(\bullet)$. The $\xi^{1 / 3}$ behavior is shown by the lines.

culated using Eq. (1) for the case of random crystallite orientations,

$$
S_{\text {poly-cryst. }}(q)=\frac{F_{D W}(q)}{4 \pi} \int_{0}^{2 \pi} d \varphi \int_{0}^{\pi} d \theta \sin (\theta) S_{\text {rhcp }}(\boldsymbol{q})
$$

with $\boldsymbol{q}=q[\sin (\theta) \cos (\varphi), \sin (\theta) \sin (\varphi), \cos (\theta)]$. To account for the decreasing intensity with increasing $q$ due to thermal motion or quenched disorder in the crystal, the Debye Waller factor, $F_{D W}(q)=\exp \left(-q^{2}\langle u\rangle^{2} / 3\right)$, is included in Eq. (2), with $\langle u\rangle$ the average displacement of the particles in the crystal from their lattice positions. The fluid contribution is approximated by a Percus-Yevick structure factor [26] that is smeared out due to the limited $q$ resolution of the instrument. As shown in Fig. 3, these two contributions give reasonable fits to the measured $S_{b g}(q)$ using $\alpha=0.5$, which corresponds to a fully random stacking of hexagonal planes. However, the data do not allow an accurate determination of the fluid and the polycrystalline contribution, as the fluid structure factor of microgels at high concentrations is not well known and only an approximate form factor was used to obtain $S_{b g}(q)$.

The monocrystalline contribution to the measurement is determined as $S_{\text {mono-cryst. }}(q)=S(q)-S_{\text {bg }}(q)$, where $S(q)$ is obtained using the mask shown in Fig. $1\left(\right.$ a). $S_{\text {mono-cryst. }}(q)$ is accounted for by considering also the rhcp model of Eq. (1), but in this case, we use a fixed crystal orientation where the hexagonal planes are perpendicular to the incident beam, such that the beam direction coincides with the direction $\hat{\boldsymbol{c}}$ of the Bragg rods,

$$
S_{\text {mono-cryst. }}(q)=\frac{F_{D W}(q)}{2 \pi} \int_{0}^{2 \pi} d \varphi S_{\text {rhcp }}(\boldsymbol{q})
$$

with $\boldsymbol{q}=q[\cos (\varphi), \sin (\varphi), 0]$ and $\hat{\boldsymbol{c}}=(0,0,1)$. As illustrated by Fig. 4 , rhcp fits with $\alpha=0.5 \pm 0.1$ to $S_{\text {mono-cryst. }}(q)$ account for all the observed peaks and even the peak intensities for all studied concentrations. For deviations $\gtrsim 0.1$ from $\alpha=0.5$, peak intensities or peak positions do not agree. In addition, we find $\alpha$ to be constant without a clear trend with $\xi$.

The value of $\langle u\rangle$ in the Debye Waller factor was adjusted to bring the fitted scattering intensity to the measured one in the range $0.0035 \leqq q \leq 0.008 \AA^{-1}$. By doing this, we find $\langle u\rangle / a=0.06 \pm 0.01$, where $a$ is the nearest-neighbor distance of the lattice sites. For hard spheres, $\langle u\rangle / a$ decreases with concentration, as the motion becomes increasingly more constrained at higher concentrations. The constancy of $\langle u\rangle / a$ observed for our microgel suspensions might reflect the unknown change of the microgel form factor with $\xi$, at high $\xi$. This uncertainty in the particle form factor is probably also responsible for the differences between the measured and the modeled intensities at $q>0.0035 \AA^{-1}$ in Figs. 3 and 4 .

The peak positions obtained from samples with different $\xi$ vary according to $\xi^{1 / 3}$, as shown in Fig. 5 for the first four peaks; only the first two could be resolved by SANS and USANS [12]. This confirms the isotropic compression of the crystal with increasing concentration without a structural change. Furthermore, the peaks found by SAXS agree well with those determined by SANS and USANS.

\section{CONCLUSIONS}

From the presented SAXS data we conclude that 2VP microgel particles crosslinked with DVB form crystals with rhcp structure even at very high concentrations, which are expected to lead to interpenetration and/or deformation of the particles. The observation of hexagonally arranged Bragg peaks allows exclusion of other crystal structures. We find that even at densities that cannot be reached with hard spheres, the studied soft particles form the same crystal structure as found in hard spheres. While all the observed peaks of the structure factor can be accounted for with the rhcp structure, the observed scattering intensity deviates from the fits in some cases for $q \gtrsim 0.0035 \AA^{-1}$. This is probably due to the form factor, which is not well known at the high concentrations used in this study. Investigations of the shrinkage, deformation, or interpenetration of microgels at high densities are needed to elucidate the role of particle shape and polydispersity in the observed crystals.

\section{ACKNOWLEDGMENTS}

We thank Benjamin Sierra-Martin for the particle synthesis. We also acknowledge financial support from the Adolphe Merkle Foundation and DPI Grant No. DPI2008-06624-C0303. Finally, we thank Andreas Menzel for his support during the SAXS measurements at the Swiss Light Source, PSI. 
[1] R. Pelton, Adv. Colloid Interface Sci. 85, 1 (2000).

[2] J. J. Lietor-Santos et al., Macromolecules 42, 6225 (2009).

[3] A. Fernandez-Nieves et al., Macromolecules 33, 2114 (2000).

[4] A. Fernandez-Nieves et al., J. Chem. Phys. 115, 7644 (2001).

[5] A. Fernandez-Nieves et al., J. Chem. Phys. 119, 10383 (2003).

[6] B. R. Saunders and B. Vincent, Adv. Colloid Interface Sci. 80, 1 (1999).

[7] D. J. Beebe et al., Nature (London) 404, 588 (2000).

[8] S. V. Vinogradov et al., Adv. Drug Delivery Rev. 54, 135 (2002).

[9] M. J. Serpe et al., Adv. Mater. 16, 184 (2004).

[10] D. M. Heyes and A. C. Branka, Soft Matter 5, 2681 (2009).

[11] D. Gottwald et al., Phys. Rev. Lett. 92, 068301 (2004).

[12] U. Gasser et al., Phys. Rev. E 79, 051403 (2009).

[13] T. Hellweg et al., Colloid Polym. Sci. 278, 972 (2000).

[14] P. S. Mohanty and W. Richtering, J. Phys. Chem. B 112, 14692 (2008).
[15] P. G. Bolhuis et al., Nature (London) 388, 235 (1997).

[16] S. Pronk and D. Frenkel, J. Chem. Phys. 110, 4589 (1999).

[17] A. Fernandez-Nieves et al., J. Chem. Phys. 120, 374 (2004).

[18] A. Loxley and B. Vincent, Colloid Polym. Sci. 275, 1108 (1997).

[19] A. Bergamaschi et al., The 16th International Workshop on Vertex Detectors (Proceedings of Science, Lake Placid, NY, 2007).

[20] U. Gasser, J. Phys.: Condens. Matter 21, 203101 (2009).

[21] B. J. Ackerson et al., Phys. Rev. E 59, 6903 (1999).

[22] S. Auer and D. Frenkel, Nature (London) 413, 711 (2001).

[23] P. Ziherl and R. D. Kamien, Phys. Rev. Lett. 85, 3528 (2000).

[24] W. Loose and B. J. Ackerson, J. Chem. Phys. 101, 7211 (1994).

[25] S. Hendricks and E. Teller, J. Chem. Phys. 10, 147 (1942).

[26] J. K. Percus and G. J. Yevick, Phys. Rev. 110, 1 (1958). 